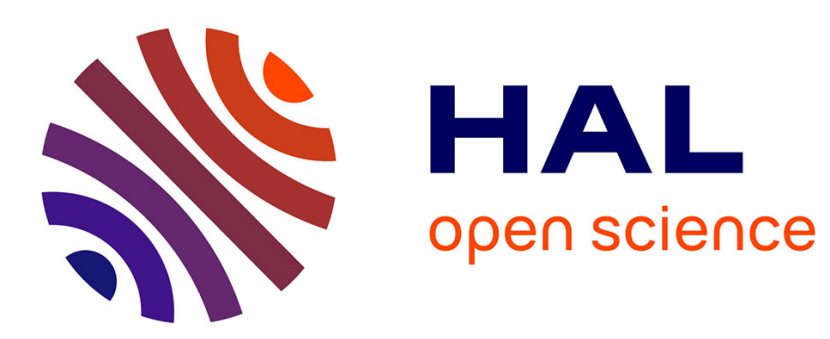

\title{
Modeling and Control of a Tilt tri-rotor Airplane
}

\author{
Duc Anh Ta, Isabelle Fantoni, Rogelio Lozano
}

\section{To cite this version:}

Duc Anh Ta, Isabelle Fantoni, Rogelio Lozano. Modeling and Control of a Tilt tri-rotor Airplane. American Control Conference (ACC 2012), Jun 2012, Montreal, Canada. pp.131-136. hal-00767341

\section{HAL Id: hal-00767341 https://hal.science/hal-00767341}

Submitted on 19 Dec 2012

HAL is a multi-disciplinary open access archive for the deposit and dissemination of scientific research documents, whether they are published or not. The documents may come from teaching and research institutions in France or abroad, or from public or private research centers.
L'archive ouverte pluridisciplinaire HAL, est destinée au dépôt et à la diffusion de documents scientifiques de niveau recherche, publiés ou non, émanant des établissements d'enseignement et de recherche français ou étrangers, des laboratoires publics ou privés. 


\title{
Modeling and Control of a Tilt tri-rotor Airplane
}

\author{
Duc Anh Ta, Isabelle Fantoni and Rogelio Lozano
}

\begin{abstract}
A helicopter offers the capability of hover, slow forward displacement, vertical take-off and landing while a conventional airplane has the performance of fast forward movement, long reach and superior endurance. The aim of this paper is to present the modelling and control of a tilt trirotor UAV's configuration that combines the advantages of both rotary wing and fixed wing vehicle.
\end{abstract}

\section{INTRODUCTION}

Nowadays, Unmanned Aerial Vehicle (UAV) possess several applications in the military and civil domains: surveillance, detection, reconnaissance, search and rescue operations... The objective of this work is to design a miniature aircraft which has the performance of an airplane for the forward flight and the capacity of a helicopter for the hover flight. In the past, this configuration has motivated lots of researchers because this type of aircraft does not require a runway and the ability of hover makes it very useful for aerial surveillance missions. In [4], ongoing works on the development of a quadrotor aerial vehicle having a tilt-wing mechanism using an LQR and a sliding mode controller for the stabilization of the attitude and the altitude are presented via vertical take-off and landing simulations. Other simulation results of the transition to level flight of a tiltwing aircraft which uses a gain-scheduled, multi-variable $H_{\infty}$ control law are obtained in [7]. More longitudinal dynamic simulation results are found in [6], in which nonlinear approaches are used for the autonomous transition of two vertical/short take-off and landing aircraft (a fixed-wing fighter equipped with a vectored thrust and lift fan and a tilt rotor). In [1], we proposed a convertible tail-sitter UAV which has the capability of transition between the vertical and horizontal flight. However, in order to make a transition, the whole fuselage of the convertible airplane must rotate to the horizontal. In this paper, we propose a configuration of the tilt-rotor mini aircraft capable of performing the hovering, transition, vertical take off and landing. In the phases of transition, the fuselage still remains in the horizontal, then the transition is more stable than the convertible airplane. The detailed mathematical model is obtained by the equations of Newton-Euler and the aerodynamic formulas. In terms of control, we propose a simple nonlinear PID control using Neural Network to stabilize the attitude and the altitude and a motion profile generator to realize the phases of take-off, landing and transition.

The paper is organized in five sections. The second section is devoted to the presentation of the platform. The mechanical structure and the aerodynamic model of the aircraft will be presented in the third section. The fourth section contains our contributions, namely the presentation

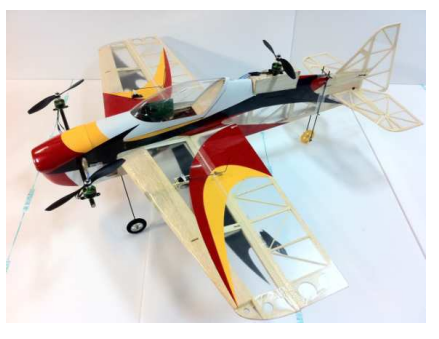

Fig. 1. Prototype of the Tilt tri-rotor Airplane

of a control strategy to achieve a longitudinal flight path. Simulation results are presented in the fifth section. Finally, the paper ends with some conclusions.

\section{TILT TRI-ROTOR AIRPLANE}

1) Platform: The tilt tri-rotor airplane has been built and completely developed at Heudiasyc laboratory (see Figure 1). It has a total weight of about $1250 \mathrm{~g}$ and uses one $2200 \mathrm{mAh}$ LiPo battery. In the vertical flight (Figure 2), the altitude is controlled by collective thrust of the three brushless electric DC Motors. The roll motion is achieved with the difference of thrust between the two front motors: the left rotates in the clockwise direction while the right in counterclockwise direction. The rear motor compensates the force generated by two front motors to stabilize the pitch angle of the airplane.

After take-off, the two front motors tilt forward thanks to an electromechanical system. The rear motor reduces its rotational speed according to the tilt angle $\delta$ of the two font motors. When $\delta$ reaches zero degree, i.e. the axis of both front motors coincides with the axis of the fuselage, the rear motor will stop completely. The airplane then flies horizontally like a conventional aircraft (Figure 3) with a cruise speed to the target. In horizontal flight, the roll motion is controlled by moving the ailerons differentially, the pitch motion is controlled by moving the elevator and the yaw motion is controlled by moving the rudder. When the vehicle reaches the target, the two front motors tilt to the vertical and the vehicle returns to the helicopter mode and lands to the ground vertically. Note that the rotational speeds of both front motors are equal in the phases of transition and horizontal flight.

2) Embedded Control System: An Inertial Measurement Unit (Microstrain, 3DM-GX3-25) is used to obtain the attitude. It weighs just $11.5 \mathrm{~g}$ and is composed of three triaxial accelerometers and angular rate gyros as well as three orthogonal magnetometers. A Digital Signal Controller (Microchip, dsPIC33FJ256GP710) was selected to implement the Embedded Control System. The measurement used for 


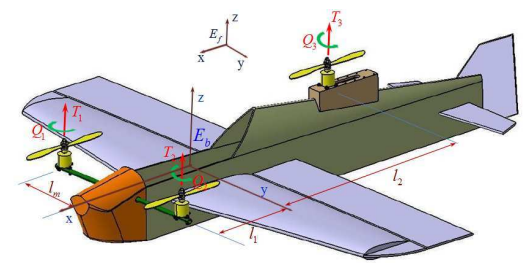

Fig. 2. Vertical flight (helicopter mode)

the altitude is obtained from the low cost ultrasonic sensor (SRF10) which has a range of $10 \mathrm{~cm}$ to $3 \mathrm{~m}$.

\section{MATHEMATICAL MODEL}

From the design model of the tilt rotor airplane described above, the Newton-Euler equations of motion describing the six degrees of freedom of the system can be separated in translational motion $\left(\Sigma_{P}\right)$ and rotational motion $\left(\Sigma_{A}\right)$ :

$$
\begin{gathered}
\Sigma_{P}:\left\{\begin{array}{l}
\dot{\vec{p}}^{f}=R \vec{v}^{b} \\
\dot{\vec{v}}^{b}=R^{T} \vec{g}^{f}+\frac{1}{\bar{m}} \vec{F}_{A, T}^{b}-\vec{\omega} \times \vec{v}^{b}
\end{array}\right. \\
\Sigma_{A}:\left\{\begin{array}{l}
\dot{\Theta}=J(\Theta) \vec{\omega} \\
I \dot{\vec{\omega}}=-\vec{\omega} \times I \vec{\omega}+\vec{\Gamma}_{A, T}
\end{array}\right.
\end{gathered}
$$

where: $R$ is the rotation matrix from $E_{b}$ to $E_{f} ; \vec{p}^{f}$ represents the position of the airplane in $E_{f} ; \vec{v}^{b}$ is the linear velocity vector presented in $E_{b} ; \bar{m}=\operatorname{diag}(m) \in \mathbb{R}^{3 \times 3}$ where $m$ $(\simeq 1250 \mathrm{~g})$ is the weight of the vehicle; $\vec{g}^{f}$ is the vector of the gravity acceleration in $E_{f} ; \vec{\omega}$ is the angular velocity vector; $\Theta=\left[\begin{array}{ccc}\phi & \theta & \psi\end{array}\right]$ is the vector of the Euler angles; $I \in$ $\mathbb{R}^{3 \times 3}$ represents the inertial matrix of the airplane; $\vec{F}_{A, T}^{b}=$ $\vec{F}_{A}^{b}+\vec{F}_{T}^{b}$ is the vector of the external aerodynamic forces and the thrusts expressed in the coordinate $E_{b} ; \vec{\Gamma}_{A, T}=\vec{\Gamma}_{A}+\vec{\Gamma}_{T}$ is the external torque vector; $J(\Theta)$ is the Jacobian matrix defined as:

$$
J(\Theta)=\left(\begin{array}{ccc}
1 & \tan \theta \sin \phi & \tan \theta \cos \phi \\
0 & \cos \phi & -\sin \phi \\
0 & \frac{\sin \phi}{\cos \theta} & \frac{\cos \phi}{\cos \theta}
\end{array}\right)
$$

Note that the singularity of the attitude representation by Euler angles can be eliminated if the airplane's orientation in space is limited. In our case, since the fuselage is always in horizontal $\left(-45^{\circ} \leqslant \theta \leqslant 45^{\circ}\right)$, the Euler angles are used to represent the attitude due to their simplicity.

At first, we analyse the forces $\vec{F}_{T}^{b}$ and the torques $\vec{\Gamma}_{T}$ generated by the actuators. Each motor produces a force $T_{i}$ parallel to its axis of rotation, and a reactive torque $Q_{i}$ opposite to the direction of rotation. Since the rotor speed reaches very high values (more than $200 \mathrm{rad} / \mathrm{sec}$ ), we can approximate the forces $T_{i}$ and the reactive torques $Q_{i}$ generated by the motors:

$$
T_{i}=b_{i} s_{i}^{2} ; Q_{i}=k_{i} s_{i}^{2}
$$

where $s_{i}$ is the rotational speed of rotor; $b_{i}$ and $k_{i}$ are two positive parameters depending on the density of air, the radius, the shape, the pitch angle of the blade and

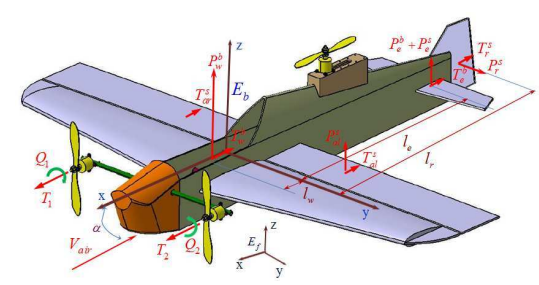

Fig. 3. Horizontal flight (airplane mode)

other factors [11]. The combination of the forces $T_{i}$ and the reactive torques $Q_{i}$ is given by:

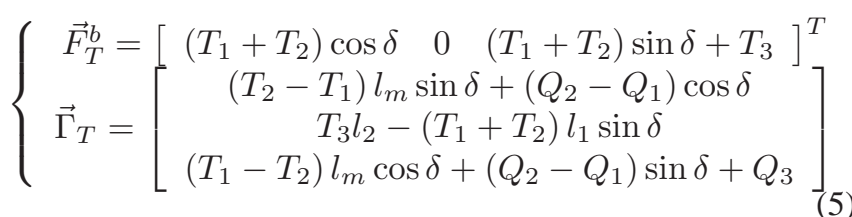

In the vertical mode (tilt angle $\delta=90^{\circ}$, Figure 2), the equation (5) becomes:

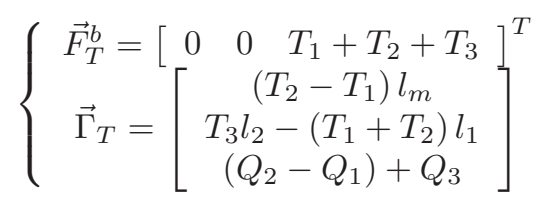

In the horizontal mode $\left(\delta=0^{\circ}, T_{3}=Q_{3}=0\right.$, Figure 3$)$, the equation (5) becomes:

$$
\left\{\begin{array}{c}
\vec{F}_{T}^{b}=\left[\begin{array}{ccc}
\left(T_{1}+T_{2}\right) & 0 & 0
\end{array}\right]^{T} \\
\vec{\Gamma}_{T}=\left[\begin{array}{lll}
\left(Q_{2}-Q_{1}\right) & 0 & \left(T_{1}-T_{2}\right) l_{m}
\end{array}\right]^{T}
\end{array}\right.
$$

The aerodynamic forces $\vec{F}_{A}^{b}$ and torques $\vec{\Gamma}_{A}$ depend on the working mode of the airplane. In all phases of flight, we assume there is no wind in the environment. So when the airplane flies vertically, there are no aerodynamic forces and torques because the speed of the vehicle is minor. However in transition and horizontal phases, the air velocity will be equal to the relative velocity of the UAV but in the opposite direction : $\vec{V}_{\text {air }}=-\vec{V}$. The vector of the air velocity creates with the airplane an angle of attack $\alpha$ (Figure 3). In these modes, the aerodynamic forces and torques are given by:

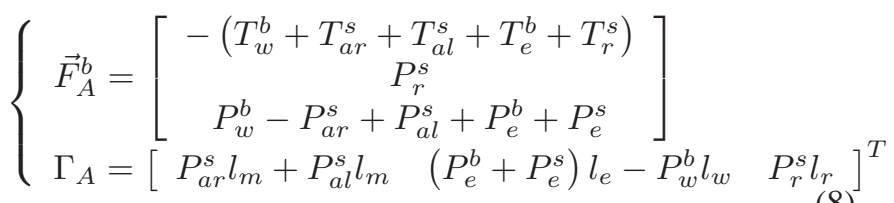

Note that in order to balance in the longitudinal dynamics, the distances $l_{e}$ and $l_{w}$ must satisfy this condition [10]:

$$
P_{e}^{b} l_{e}=P_{w}^{b} l_{w} \rightarrow \frac{l_{e}}{l_{w}}=\frac{P_{w}^{b}}{P_{e}^{b}} \approx \frac{P_{w}}{P_{e}} \approx \frac{S_{w}}{S_{e}}
$$

Then:

$$
(8) \rightarrow\left\{\begin{array}{l}
\vec{F}_{A}^{b} \approx\left[\begin{array}{lll}
-\left(T_{w}^{b}+T_{e}^{b}\right) & 0 & P_{w}^{b}+P_{e}^{b}
\end{array}\right]^{T} \\
\Gamma_{A} \approx\left[\begin{array}{lll}
P_{a r}^{s} l_{m}+P_{a l}^{s} l_{m} & P_{e}^{s} l_{e} & P_{r}^{s} l_{r}
\end{array}\right]^{T}
\end{array}\right.
$$

where $P_{i}^{s}=\frac{1}{2} \rho v_{i n}^{2} S_{i}^{s} C_{i}^{P_{\delta}}$ and $T_{i}^{s}=\frac{1}{2} \rho v_{i n}^{2} S_{i}^{s} C_{i}^{T_{\delta}}$ are respectively the lift force and the drag force created by 
slipstream with $i=a l$, ar, $e, r$ (left aileron, right aileron, elevator and rudder); $\rho$ is the density of air; $C_{i}^{P_{\delta}}=C_{i_{\delta}}^{P} \delta_{i}$; $C_{i}^{T_{\delta}}=C_{i_{\delta}}^{T} \delta_{i} ; \delta_{i}$ and $S_{i}$ are the deflection angle and the area of control surface; $v_{i n}=\sqrt{\frac{2 T_{i}}{\rho A_{\mathrm{M}}}+V^{2}}$ is the air velocity created by the propeller (inflow or induced velocity).

We have used the same analysis as in [1] and aerodynamics formulas in [9].

Then, in the vertical mode $\left(\delta=90^{\circ}\right)$ the external torque vector $\Gamma_{A, T}$ is given by:

$$
\vec{\Gamma}_{A, T_{V}}=\vec{\Gamma}_{T}=\left[\begin{array}{c}
b\left(s_{2}^{2}-s_{1}^{2}\right) l_{m} \\
b s_{3}^{2} l_{2}-b\left(s_{1}^{2}+s_{2}^{2}\right) l_{1} \\
k\left(s_{2}^{2}-s_{1}^{2}\right)+k s_{3}^{2}
\end{array}\right]
$$

In the transition mode, the external torque and external force depend on several aerodynamic parameters. Due to space limitations, the expressions are given in [2].

\section{CONTROL STRATEGY}

In the longitudinal dynamics, the tilt-rotor airplane performs the following phases of flight: vertical take-off, hover mode, transition to the horizontal, horizontal flight, transition to the vertical and vertical landing.

\section{A. Control Law}

1) Altitude Control: The control of the altitude is used when the airplane flies vertically but it is not used in the transition and horizontal flight. Extracting the translational motion on the $z$ axis of $E_{f}$ from equation (1) yields:

$$
m \ddot{z}=T \cos \theta \cos \phi-m g
$$

where $T$ is the total thrust force of the three motors. A simple PD control can guarantee the convergence of the altitude to the desired altitude $z_{d}$ :

$$
T=T_{1}+T_{2}+T_{3}=\frac{k_{p}^{z}\left(z_{d}-z\right)+k_{d}^{z}\left(\dot{z}_{d}-\dot{z}\right)+m g}{\cos \theta \cos \phi}
$$

but for security reasons and in order to improve the performance, a PID control with a saturation is used:

$$
\begin{gathered}
T=\frac{\operatorname{sat}_{T}\left(k_{p}^{z}\left(z_{d}-z\right)+k_{d}^{z}\left(\dot{z}_{d}-\dot{z}\right)+k_{i}^{z} \int\left(z_{d}-z\right)\right)+m g}{\cos \theta \cos \phi} \\
T=\frac{\operatorname{sat}_{T}\left(k_{p}^{z} e_{p}^{z}+k_{i}^{z} e_{i}^{z}+k_{d}^{z} e_{d}^{z}\right)+m g}{\cos \theta \cos \phi}
\end{gathered}
$$

where $\operatorname{sat}_{M}(x)$ is a nonlinear saturated sigmoid function:

$$
\operatorname{sat}_{M}(x)=\frac{M\left(1-e^{-2 x / M}\right)}{\left(1+e^{-2 x / M}\right)}
$$

Note that $\lim _{x \rightarrow \pm \infty}\left(\operatorname{sat}_{M}(\mathrm{x})\right)=M \operatorname{sign}(x)$, where $\operatorname{sign}(x)$ is the signum function defined as $\operatorname{sign}(x)=$ $\{1$ if $x \geqslant 0 ;-1$ if $x<0\}$, then $M$ is the boundary saturation. The sigmoid function $\operatorname{sat}_{M}(x)$ becomes linear when $M$ goes to infinity.
2) Attitude Control: The attitude control is used to stabilize the UAV when it takes off and lands vertically. However in the transition phase, two front motors must tilt forward with the same rotational speed. So only the pitching movement is stabilized in the transition phases, the rolling and yawing motions are controlled manually. The bounded attitude control PID is used:

$$
\Gamma_{j}=\operatorname{sat}_{\Gamma_{j}}\left(k_{p}^{j} e_{p}^{j}+k_{i}^{j} e_{i}^{j}+k_{d}^{j} e_{d}^{j}\right)
$$

with $j=\phi, \theta, \psi$ and $\Gamma_{j}$ are the boundary of the control torques around the axes $x, y, z$.

3) Adaptative Control Using Neural Network: A modification of control parameters for PID control must be adjusted to realize more accurate control. It is obvious that the PID control method is limited because the parameters $k_{p}, k_{d}, k_{i}$ are constant. This means that the control output is not adaptable and optimal in any case. An intelligent adaptative control, which has the adaptability of control parameters to minimize the position error, is used. With the capacity of learning and adaptability of neural network, the controller can solve these problems. The parameters PID will be tuned adaptively and optimally in order to minimize the position error with respect to external perturbations.

Figure 4 shows the structure of the PID control using neural network for one degree of freedom. Here we take the pitch angle for example, the control method will be applied similarly for other degrees of freedom (altitude, roll and yaw). The block diagram of neural network is shown in Figure 5. Here, $k_{p}, k_{d}, k_{i}, e_{p}, e_{i}$ and $e_{d}$ are proportional, integral and derivative gains, the system error between desired and actual output, the integral of the system error and the difference of the system error, respectively. Neural networks are trained by the conventional back propagation algorithm to minimize the system error between the desired and actual output. In Figure 5, the input signal of the sigmoid function in the output layer becomes:

$$
x(k)=k_{p}^{\theta}(k) e_{p}^{\theta}(k)+k_{i}^{\theta}(k) e_{i}^{\theta}(k)+k_{d}^{\theta}(k) e_{d}^{\theta}(k)
$$

where,

$$
\left\{\begin{array}{l}
e_{p}^{\theta}(k)=\theta_{d}(k)-\theta(k) \\
e_{i}^{\theta}(k)=\sum_{n=1}^{k} e_{p}^{\theta}(n) \Delta T \\
e_{d}^{\theta}(k)=\frac{e_{p}^{\theta}(k)-e_{p}^{\theta}(k-1)}{\Delta T}
\end{array}\right.
$$

$\Delta T$ is the sampling time, $k$ is the discrete sequence; $\theta_{d}(k)$ and $\theta(k)$ are the desired and actual pitch angle. To tune the gains of the PID controller $k_{p}, k_{i}, k_{d}$, the Gradient Descent method using the following equation was applied:

$$
k_{j}^{\theta}(k+1)=k_{j}^{\theta}(k)-\eta_{j}^{\theta} \frac{\partial E(k)}{\partial k_{j}^{\theta}}
$$

for $j=p, i, d$ where $\eta_{p}^{\theta}, \eta_{i}^{\theta}, \eta_{d}^{\theta}$ are learning rates determining the convergence speed, and $E(k)$ is a cost function. The PID parameters are updated at the step $k$ in order to decrease the cost function. Since the control purpose is to decrease the 


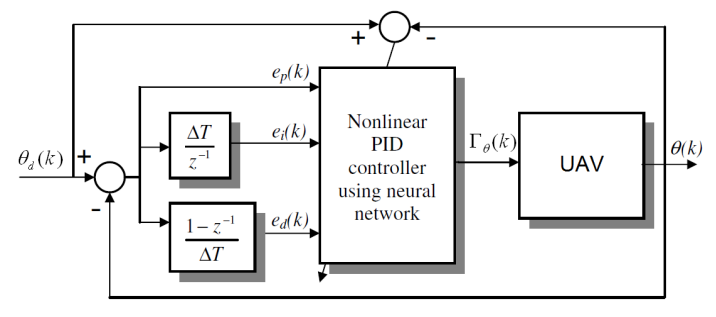

Fig. 4. Structure of the nonlinear PID controller using neural network.

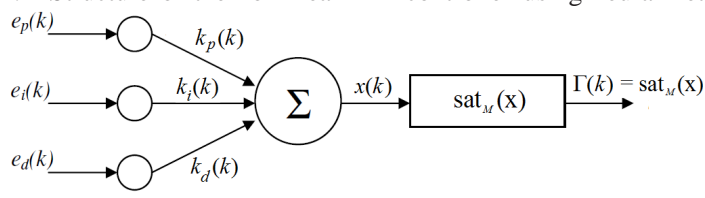

Fig. 5. Block diagram of neural network.

error $e_{p}^{\theta}(k)$ therefore we choose the cost function $E(k)=$ $\frac{1}{2}\left(\theta_{d}(k)-\theta(k)\right)^{2}$. According to [3], the parameters are updated for $j=p, i, d$ :

$$
k_{j}^{\theta}(k+1)=k_{j}^{\theta}(k)+\eta_{j}^{\theta} e_{j}^{\theta}(k) e_{j}^{\theta}(k) \frac{\partial \Gamma_{\theta}(k)}{\partial x}
$$

where :

$$
\begin{aligned}
& \frac{\partial \Gamma_{\theta}(k)}{\partial x}=\frac{\partial\left(\operatorname{sat}_{\Gamma_{\theta}} x(k)\right)}{\partial x} \\
& =\frac{\partial}{\partial x}\left(\frac{\Gamma_{\theta}\left(1-e^{-2 x(k) / \Gamma_{\theta}}\right)}{\left(1+e^{-2 x(k) / \Gamma_{\theta}}\right)}\right)=\frac{4 e^{-2 x(k) / \Gamma_{\theta}}}{\left(1+e^{-2 x(k) / \Gamma_{\theta}}\right)^{2}}
\end{aligned}
$$

Then the PID parameters are tuned at every loop.

We can notice that when the learning rates $\eta_{j}$ (with $j=$ $p, i, d)$ are minor, the convergence speed will be slow, which will decrease the adaptation of system. But if a high $\eta_{j}$ is used, the Neural Network adapts more quickly but the cost function will overpass more easily the minimum value and the gains cannot converge to the needed values. In order to improve the Gradient Descent algorithm, the learning rates $\eta_{j}$ are also updated adaptively as follow:

$$
\eta_{j}(k+1)=\left(1+\varsigma_{j} \operatorname{sign}(E(k+1)-E(k))\right) \eta_{j}(k)
$$

where $\varsigma_{j}$ are positive parameters.

\section{B. Trapezoidal Trajectory of Velocity - Servo Control}

In the case when the vehicle autonomously takes off, lands and makes a transition, the controller requires the next desired altitude and the next desired tilt angle. Then a trajectory generation algorithm must be used for optimum motion control. We have used the same motion profile algorithm that controls the speed and acceleration as in [1].

The trajectory generator will produce trapezoidal shaped velocity curves for a long movement and triangular curves for a short movement where maximum velocity was not reached.

The control law of the altitude will force the vehicle to track the new desired altitude created by the trajectory generator in order to realize the process of take-off and landing. Figure 6 shows the rotor's tilt angle generated by the motion profile algorithm and its adaptation when the airplane makes transition to the horizontal flight.

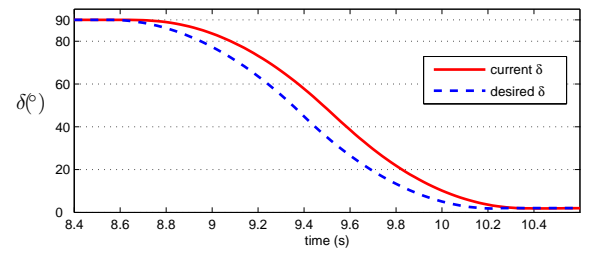

Fig. 6. Desired titlt angle generated by the motion profile algorithm and its adaptation

During the transition to the level flight when both front rotors start tilting forward, the force of motors on the $x$ axis appears and then the aircraft speed increases. The aerodynamic lift force appears, however we can not measure or estimate this force. So the control law of the pitching movement must be used. From (5) the pitching moment equation is:

$$
\Gamma_{C_{\theta}}=T_{3} l_{2}-\left(T_{1}+T_{2}\right) l_{1} \sin \delta
$$

where $\Gamma_{C_{\theta}}$ is the pitching torque deduced from the attitude control law to stabilize the aircraft, $T_{1}=T_{2}$ are the thrusts of two front motors which are given by the operator via $\mathrm{R} / \mathrm{C}$ receiver. By using this method, the operator can adjust the UAV's speed during the transition. Knowing that the tilt angle $\delta$ decreases from $90^{\circ}$ to $0^{\circ}$, so the third motor's speed must vary according to the equation:

$$
T_{3}=\frac{\Gamma_{C_{\theta}}+\left(T_{1}+T_{2}\right) l_{1} \sin \delta}{l_{2}}
$$

While the force generated by the motors in the $z$ axis decreases $\left(T_{3}\right.$ and $\left.\left(T_{1}+T_{2}\right) \sin \delta\right)$, the force in the $x$ axis will increase. Therefore the aerodynamic force $F_{A}^{z}$ at the moment will increase in order to compensate the reduction of $F_{T}^{z}$ thanks to the high velocity of the vehicle.

\section{Simulation Results}

\section{A. Longitudinal Flight Simulation}

In this section, simulation results from Matlab-Simulink and MSC. Visual Nastran 4D simulator are presented to demonstrate the performance of the control strategy. In this simulation, we assume that the airplane is stable in lateral dynamics and the reaction of the tilt angle's servo motor is much faster than the vehicle's dynamic. It means that we exam here only the longitudinal dynamics and the tilt angle's servo adapts immediately with respect to the desired tilt angle. According to the logics of transition $(1=$ transition to level flight, $0=$ transition to hover) and of vertical flight ( 1 $=$ take off, $0=$ landing $)($ Figure 8$)$, we can divide the phases of flight as follows:

(1). Take-off (from the beginning to second 3): Initially, the vehicle takes off automatically up to $2 \mathrm{~m}$, the altitude velocity has a trapezoidal form, it accelerates and reduces the speed when the vehicle reaches $2 \mathrm{~m}$.

(2). Transition to the level flight (from second 3.5 to 8.5): the airplane makes the transition in second 3.5, the tilt angle varies from $90^{\circ}$ to $0^{\circ}$ and the pitch angle inclines $2^{\circ}$ in 


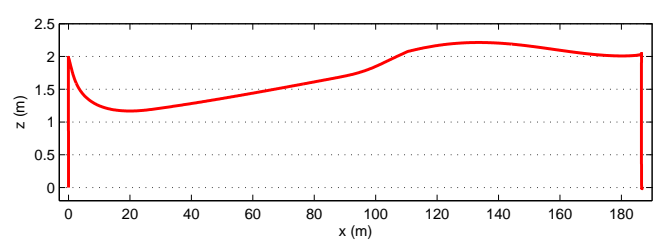

Fig. 7. Longitudinal flight path

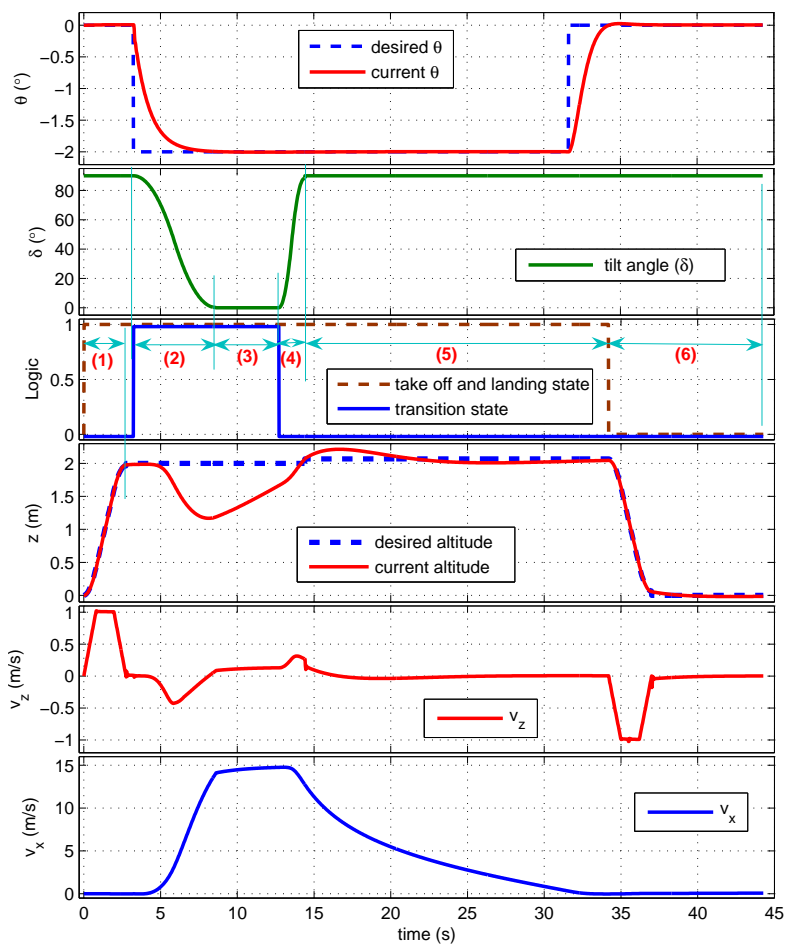

Fig. 8. State variables of the airplane during the period of flight

order to have a high angle of attack. This pitch angle can be increased more but $2^{\circ}$ is sufficient to prove the performance of the control strategy. The vehicle loses the altitude at the beginning of the transition because its low speed cannot permit to create the sufficient lift force. In fact, we can increase the rotational speed of both front rotors so that the airplane can avoid losing the altitude during the transition but here they are still kept at the value ( $349 \mathrm{rad} / \mathrm{s})$ at which they contribute to the total thrust force equal to the airplane's weight in the vertical flight (Figure 10). During the transition, the rotation speed of the rear motor reduces to $0 \mathrm{rad} / \mathrm{s}$ according to the equation (25) and the airplane accelerates thanks to the increase of thrust force $F_{T}^{x}$ (see Figure 9). The transition terminates at second 8.3 when the tilt angle reaches $0^{\circ}$.

(3). Horizontal flight (from second 8.5 to 12.5): After the transition, the velocity still increases to a certain speed although the rotational speed of the both front motors has been reduced to $270 \mathrm{rad} / \mathrm{s}$ (see Figure 10). The velocity then converges to a constant value due to the friction drag. It means that at the moment the longitudinal acceleration of the airplane is zero. In Figure 11, we can notice that

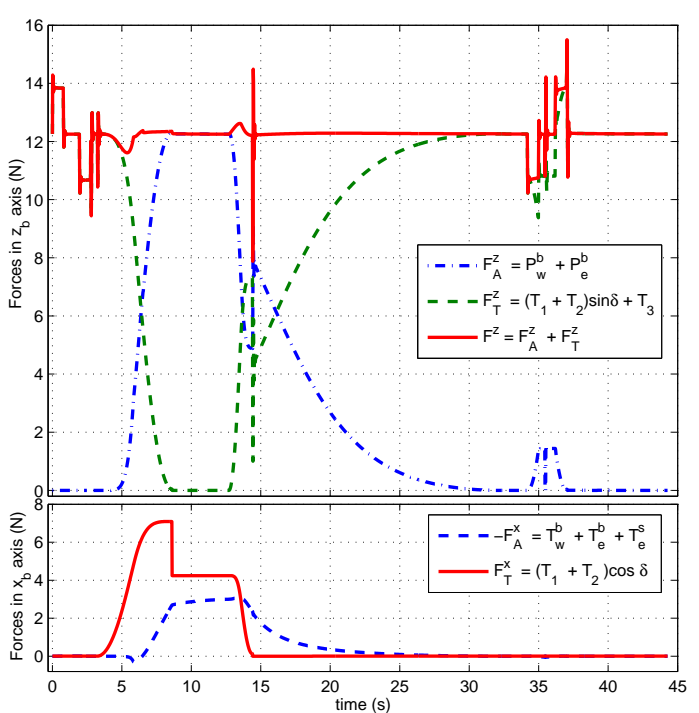

Fig. 9. Forces generated in $E_{b}$ axis

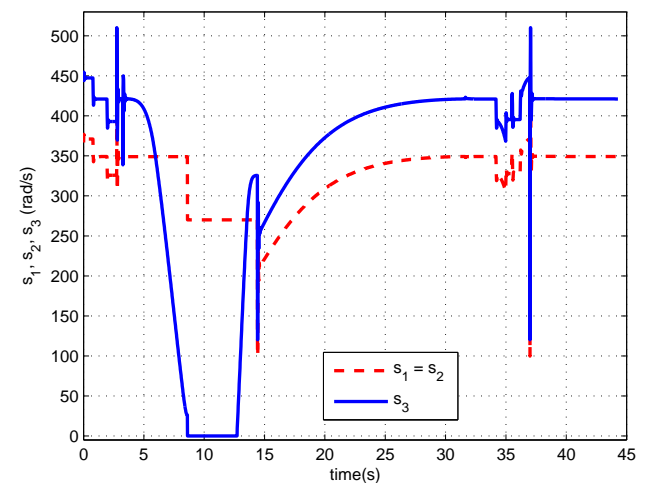

Fig. 10. Speed of each rotor

the angle of attack also converges to a constant value. Despite this small value (approximately $1.5^{\circ}$ ), it is enough to create the lift force thanks to the high speed of the aircraft (approximately $15 \mathrm{~m} / \mathrm{s}$ )(note that the nominal airplane's speed is about $13 \mathrm{~m} / \mathrm{s}$ ).

(4). Transition to the hover flight (from second 12.5 to 14.5): During the transition, the rear rotor increases the speed from zero to a necessary value in order to maintain the pitch angle at $-2^{\circ}$ while the speed of both front rotors still remains at $270 \mathrm{rad} / \mathrm{s}$. The velocity of the airplane decreases because the force $F_{T}^{x}$ reduces rapidly (Figure 9 ). Therefore the aerodynamic lift force $F_{A}^{z}$ also decreases while the total thrust force of motors $F_{T}^{z}$ increases.

(5) and (6). Vertical flight and landing (from second 14.5 to 43): Without the $F_{T}^{x}$, the aircraft still flies with cruise speed because it still has the momentum in horizontal flight. However its velocity reduces because of the drag force and the pitch angle $-2^{\circ}$. The airplane still has the lift force $F_{A}^{z}$ (Figure 9) thanks to its velocity but this lift force also reduces to zero according to the velocity. When the velocity reaches zero, the desired pitch angle is zero to stabilize the airplane 


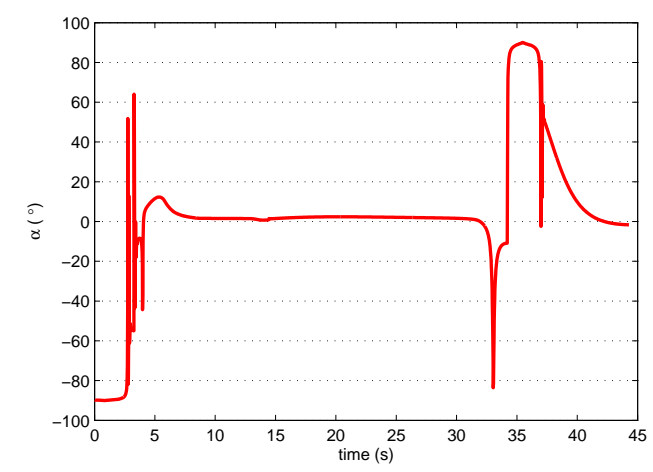

Fig. 11. Angle of attack

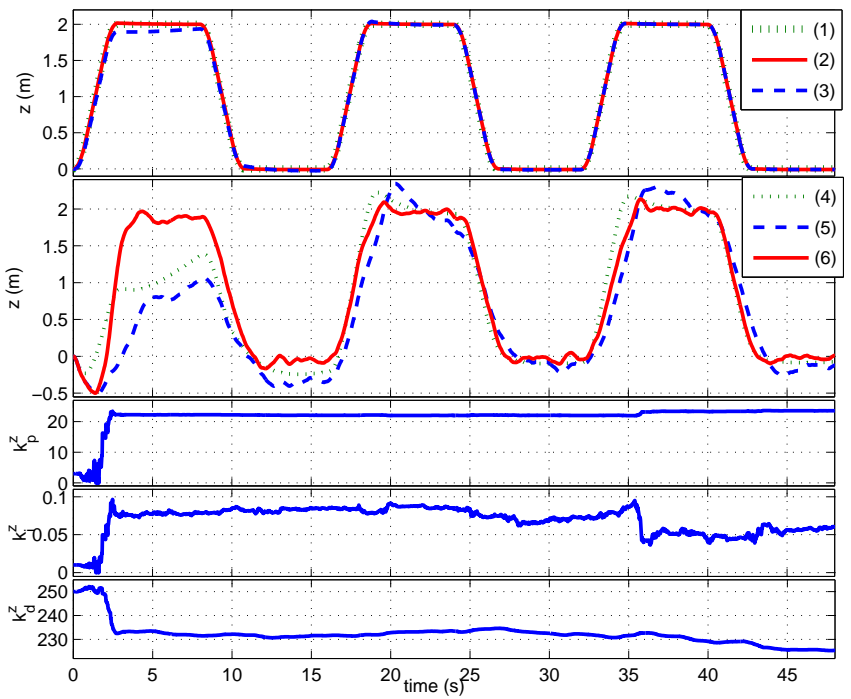

Fig. 12. Control improved by Neural Network

and after that, the airplane lands to the ground at the second 34.

Note that during the period of flight, the sum of the aerodynamic lift force $F_{A}^{z}$ and the total thrust force $F_{T}^{z}$ is always approximate to the weight of the vehicle $(m g \approx$ $12.26 \mathrm{~N})$ (Figure 9). It guarantees that the airplane cannot fall down. The control law of the altitude is inactivated during the horizontal flight and the transition, the profile of the altitude velocity is trapezoidal in the periods of take-off and landing and there is no overshooting.

\section{B. Control performance improved by Neural Network}

The Figure 12 shows that the performance of the control was improved by Neural Network when the vehicle realizes 3 times of take-off and landing from $\mathrm{Om}$ to $2 \mathrm{~m}$. The curve (1) is the desired position generated by the generator of trajectory and the second one (2) is the adaptation of altitude control when the gains were chosen correctly. The curve (3) is the adaptation when the gains were chosen incorrectly. The adaptation is almost acceptable thanks to the trajectory generator but there are static errors at the beginning. However these static errors are eliminated quickly by the integral control. In the curve (4), the offset of the altitude control $m g$ in the equation (15) was changed to 0.6mg (wrong estimation of system parameters) and the gains still remain incorrect like the case (3). The parameters of control in the test (5) are similar as (4), but here sensor noises and external disturbances (zero mean, bounded noise processes with standard deviations of $\sigma_{z}=0.03 \mathrm{~m}$ and $\sigma_{F z}=4 \mathrm{~N}$ ) were added to altitude's measure and $F^{z}$ respectively. The conditions of test (6) are the same as (5) (wrong gains and parameters of control, input and output perturbations) but the gains were now corrected adaptively by Neural Network.

\section{CONCLUSIONS AND FUTURE WORKS}

In this work, we have presented the dynamic model of a tilt tri-rotor airplane and a simple control strategy to achieve a longitudinal flight path. The obtained simulation results demonstrate that the proposed control strategy could be implemented in reality. The control law is simple and suitable for embedded applications, it does not require a high computational cost for control loop. The proposed approach is currently implemented on the platform and the experimental tests will be presented in a longer version of the paper.

\section{ACKNOWLEDGMENTS}

This work has been supported by the French Armament Procurement Agency (DGA) and the French National Center for Scientific Research (CNRS).

\section{REFERENCES}

[1] D. A. Ta, I. Fantoni, R. Lozano, Modeling and Control of a Convertible Mini-UAV, in 18th World Congress of the International Federation of Automatic Control, Italy, 2011.

[2] D. A. Ta, Avion convertible à décollage et atterrissage vertical, $P h D$ thesis, University of Technology of Compiègne, France, December 2011.

[3] T.D.C. Thanh and K.K. Ahn, Techical note: Nonlinear PID control to improve the control performance of 2 axes pneumatic artificial muscle manipulator using neural network, In Journal of Mechatronics, 2006.

[4] K. T. Oner, E. Cetinsoy, E. Sirimoglu, C. Hancer, T. Ayken and M. Unel, LQR and SMC Stabilization of a New Unmanned Aerial Vehicle, in World Academy of Science, Engineering and Technology, 2009.

[5] F. Kendoul, I. Fantoni, R. Lozano, Modeling and control of a small autonomous aircraft having two tilting rotors, in Proceedings of the 44th IEEE Conference on Decision and Control, and the European Control Conference, Spain, 2005.

[6] Y. Xili, F. Yong and Z. Jihong, Transition Flight Control of Two Vertical/Short Takeoff and Landing Aircraft, in Journal of Guidance, Control and Dynamics, vol.31, no.2, 2008.

[7] J. Dickeson, D. Miles, O. Cifdaloz, V. Wells and A. A. Rodriguez, Robust LPV $H_{\infty}$ Gain-Scheduled Hover-to-Cruise Conversion for a Tilt-Wing Rotorcraft in the Presence of CG Variations, in Proceedings of the 2007 American Control Conference, USA, 2007.

[8] R. Lozano, editor. Unmanned Aerial Vehicles. John Wiley, 2010.

[9] B. W. Mc Cormick, Aerodynamics Aeronautics and Flight Mechanics, John Wiley and Sons Inc, 1995.

[10] Michael V.Cook, Flight Dynamics Principles: A Linear Systems Approach to Aircraft Stability and Control, Elsevier, 2007.

[11] G. Fay, Derivation of the aerodynamic forces for the mesicopter simulation, Technical Report, Stanford University, USA, 2001.

[12] A. R. Teel, Global stabilization and restricted tracking for multiple integrators with bounded controls, Systems and Control Letters, 18:165171, 1992.

[13] T.D.C. Thanh and K.K. Ahn, Intelligent Phase Plane Switching Control of Pneumatic Artificial Muscle Manipulators with Magneto Rheological Brake, Journal of Mechatronics, vol. 16, no. 2, 2006. 